\title{
Revealing Embedded Crystals through their Diffracting Signals in Transmission Electron Microscopy
}

\author{
E.F. Rauch*, M. Véron \\ Univ. Grenoble Alpes, CNRS, Grenoble INP, SIMAP, 38000 Grenoble, France \\ * Corresponding author: edgar.rauch@simap.grenoble-inp.fr
}

While phases and crystallographic orientation are routinely sorted and recognized using their electron diffraction signature in a transmission electron microscope (TEM), these facilities are still strongly limited in case of precipitates embedded in a crystallized matrix. In transmission, the electron beam crosses the whole thickness of the sample and collect the diffracting signal of all overlapping grains or phases. When nanoparticles are considered, the resulting diffraction patterns are mainly composed of the dominant matrix reflections plus some additional - frequently faint - information related to the second phase. This leads to frequent misindexing when phase or orientation are recognized with automated tools.

However, the precipitates signatures being contained in the diffraction patterns they can be extracted with relevant procedures. In particular when 4D-SPED datasets are considered, numerical approaches may be considered to by-pass these limitations. 4D-SPED refers to the collection of all successive 2D Electron Diffraction patterns acquired in Precession mode while the nano electron beam is Scanning a $2 \mathrm{D}$ area.

Two types of such post-processing algorithms were developed and applied to the case of a Custom 465® precipitation hardened stainless steel containing $\eta-\mathrm{Ni} 3 \mathrm{Ti}$ hcp phase in Burgers orientation relationship with the martensitic matrix. A standard indexing procedure fails in retrieving either the presence or the proper orientation of the precipitates (Fig.1).

(i) The first strategy consists in emphasizing the second phase particles with Virtual Dark-Field (VDF) images. The main advantage with respect to classical dark-field images is that the 'numerical aperture' used in VDF offers full flexibility [1]. In particular, instead of using a single 'hole', a theoretical diffraction pattern (a template) representative of the orientation of one particular family of the precipitates variants was used to filter the data and to locate all the members of this family in figure 2 .

(ii) The second approach mimics the dissolution of the matrix pertaining in extraction replication by removing numerically all the matrix signal from the dataset. Either an average diffraction patterns, in which the precipitates contribution is diluted, is subtracted from each successive pattern or the matrix reflections are firstly recognized through template matching and then erased before re-indexing the full dataset as proposed in [2] (Fig. 3).

Because of the known orientation relationship, the validity of the results may be inferred in all cases by comparing the matrix $\{110\}$ pole figure (resp. $\{111\}$ ) to the $\eta$-Ni3Ti $\{0001\}$ pole figure (resp. $\{11-20\}$ ). For the present case, the subtraction strategy turns out to be more efficient because not only the location but also the crystallographic orientations of the precipitates are deduced from the results, while the orientations have been used as inputs to generate the templates for the virtual dark-field construction. 


\section{References:}

[1] E. F. Rauch and M. Véron, "Virtual dark-field images reconstructed from electron diffraction patterns,” Eur. Phys. J. Appl. Phys., vol. 66, no. 1, (2014) p. 10701,.

[2] A. Valery, E. F. Rauch, L. Clément, and F. Lorut, "Retrieving overlapping crystals information from TEM nano-beam electron diffraction patterns,” J. Microsc., vol. 268, no. 2, (2017)

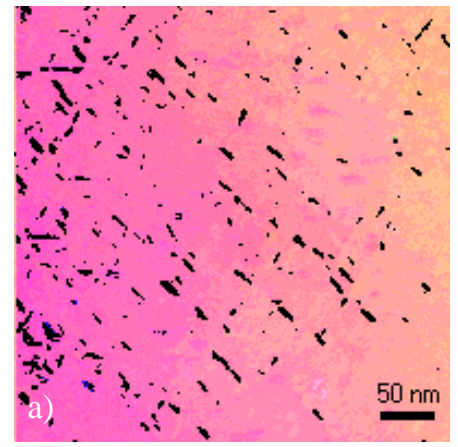

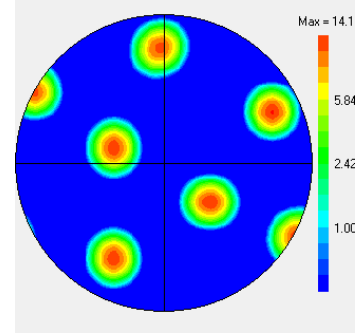

b) Matrix $\{110\}$ pole figure

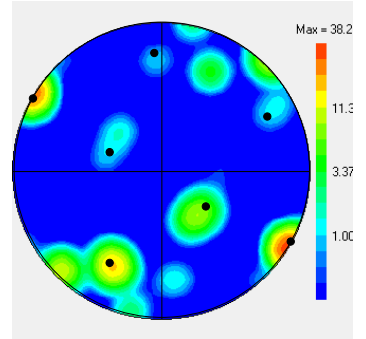

c) $\mathrm{Ni}_{3} \mathrm{Ti}\{0001\}$ pole figure

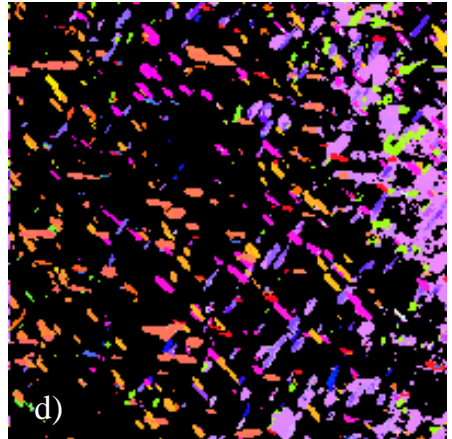

Figure 1. Orientation maps and pole figures of a-b) the Fe matrix and c-d) the $\eta-\mathrm{Ni}_{3} \mathrm{Ti}$ phase solely. The eta orientation map is obtained by disregarding the matrix signal and imposing a threshold in the correlation index (Index > 300). The expected correspondence between (0001)hcp and (110)bcc planes reproduced as superimposed black dots in (c) - is only partly obtained.
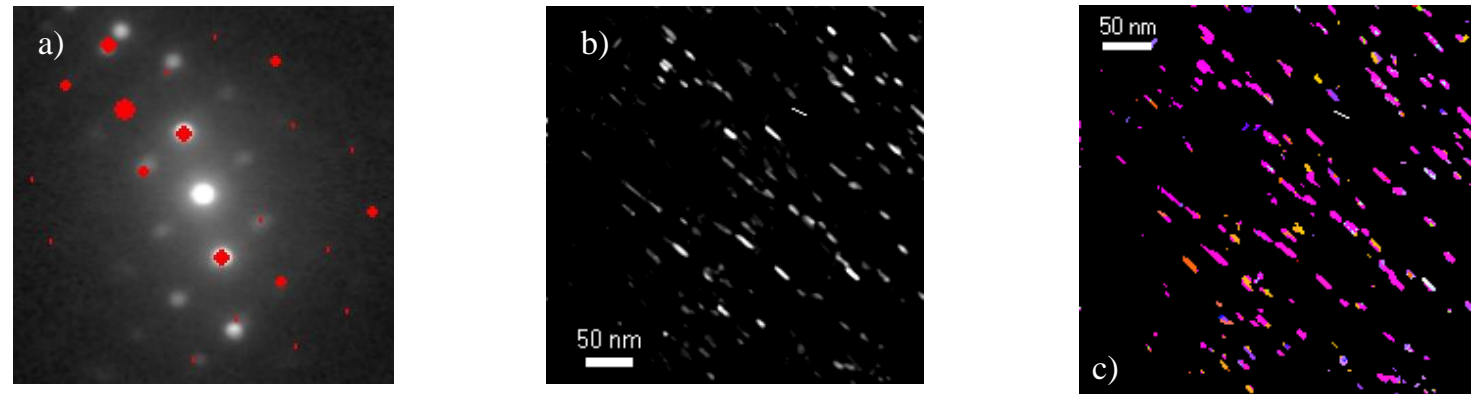

Figure 2. Ni3Ti precipitates highlighted with virtual dark-field images. In a) the numerical aperture is constructed by taking the inverse video of a variant template and produces b) a template related virtual dark-field image. The latter is used in (c) as a mask on figure 1.c to sort all the members of this family of precipitates.

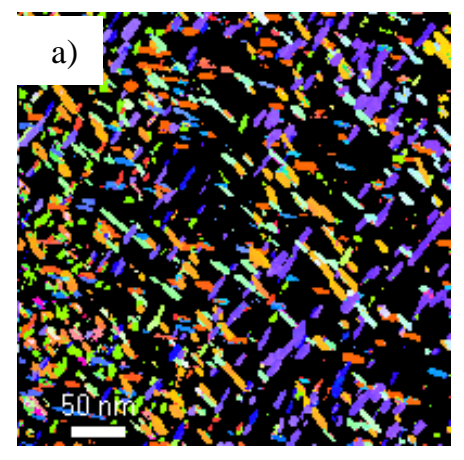

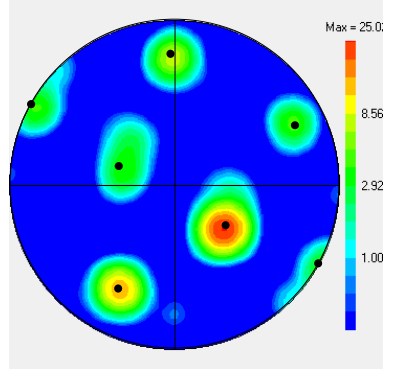

b) Ni3Ti $\{0001\}$ pole figure with superimposed $\{110\}$ matrix poles

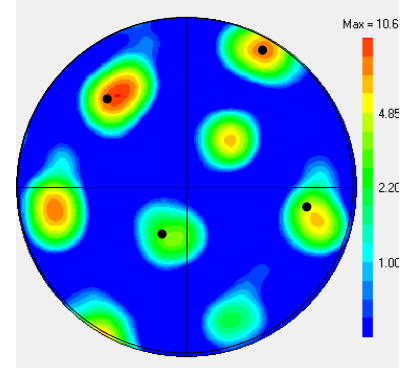

c) Ni3Ti $\{11-20\}$ pole figure with superimposed $\{111\}$ matrix pole

Figure 3. Results of the multi-indexing strategy. Precipitates a) orientation map and b-c) poles figures. Note the correspondence with the matrix related poles marked in black in the pole figures. 